\title{
Implementation of the IPACK (Infiltration between the Popliteal Artery and Capsule of the Knee) block into a multimodal analgesic pathway for total knee replacement
}

\author{
Brandon Kandarian ${ }^{1}$, Pier F. Indelli $i^{2,3}$, Sanjay Sinha ${ }^{4}$, Oluwatobi O. Hunter ${ }^{5}$, \\ Rachel R. Wang ${ }^{1,5}$, T. Edward Kim ${ }^{1,5}$, Alex Kou ${ }^{1,5}$, and Edward R. Mariano ${ }^{1,5}$ \\ ${ }^{1}$ Department of Anesthesiology, Perioperative and Pain Medicine, Stanford University School of Medicine, Stanford, \\ $C A,{ }^{2}$ Department of Orthopedic Surgery, Stanford University School of Medicine, Stanford, CA, ${ }^{3}$ Orthopedic Surgery \\ Section, Surgical Service, Veterans Affairs Palo Alto Health Care System, Palo Alto, CA, ${ }^{4}$ Department of Anesthesiology, \\ St. Francis Hospital and Medical Center, Hartford, CT, ${ }^{5}$ Anesthesiology and Perioperative Care Service, Veterans Affairs \\ Palo Alto Health Care System, Palo Alto, CA, USA
}

Background: The Infiltration between the Popliteal Artery and Capsule of the Knee (IPACK) block is a new anesthesiologist-administered analgesic technique for controlling posterior knee pain that has not yet been well studied in total knee arthroplasty (TKA) patients. We compared pain outcomes in TKA patients before and after implementation of the IPACK with the hypothesis that patients receiving IPACK blocks will report lower pain scores on postoperative day (POD) 0 than non-IPACK patients.

Methods: With Institutional Review Board approval, we retrospectively reviewed data for consecutive TKA patients by a single surgeon 4 months before (PRE) and after (POST) IPACK implementation. All TKA patients received adductor canal catheters and peri-operative multimodal analgesia. The primary outcome was pain on POD 0 . Other outcomes were daily pain scores, opioid consumption, ambulation distance, length of stay, and adverse events within 30 days.

Results: Post-implementation, 48/50 (96\%) of TKA patients received an IPACK block, and they were compared with 32 patients in the PRE group. On POD 0, the lowest pain score (median [10th-90th percentiles]) was significantly lower for the POST group compared to the PRE group (0 [0-4.3] vs. 2.5 [0-7]; $\mathrm{P}=0.003)$. The highest patient-reported pain scores on any POD were similar between groups with no differences in other outcomes.

Conclusions: Within a multimodal analgesic protocol, addition of IPACK blocks decreased the lowest pain scores on POD 0. Although other outcomes were unchanged, there may be a role for new opioid-sparing analgesic techniques, and changing clinical practice change can occur rapidly.

Keywords: Adductor canal catheter; Implementation; IPACK; Local infiltration analgesia; Multimodal analgesia; Total knee arthroplasty.

Corresponding author: Edward R. Mariano, M.D., MAS

Anesthesiology and Perioperative Care Service, Veterans Affairs Palo Alto Health Care System, 3801 Miranda Avenue (112A), Palo Alto, CA 94304, USA

Tel: 1-650-849-0254, Fax: 1-650-852-3423, Email: emariano@stanford.edu

ORCID: https://orcid.org/0000-0003-2735-248X

Received: December 5, 2018. Revised: January 17, 2019. Accepted: February 14, 2019.

Korean J Anesthesiol 2019 June 72(3): 238-244

https://doi.org/10.4097/kja.d.18.00346

(c) This is an open-access article distributed under the terms of the Creative Commons Attribution Non-Commercial License (http://creativecommons.org/ licenses/by-nc/4.0/), which permits unrestricted non-commercial use, distribution, and reproduction in any medium, provided the original work is properly cited. 


\section{Introduction}

Regional analgesia for patients undergoing total knee arthroplasty (TKA) has evolved over the past 3 decades to become more selective to the operative site and balance the goals of effective pain management with early rehabilitation [1]. Local infiltration analgesia (LIA) involves local anesthetic administration by the surgeon in close proximity to the operative site and has proven efficacious [2,3]. When combined, LIA and peripheral nerve blockade provide superior pain control when compared to either approach alone $[4,5]$. Therefore, this combination has been integrated into a multimodal analgesic clinical pathway for TKA patients at our institution with extremely high adherence rates [6]. This pathway is managed by a dedicated regional anesthesiology and acute pain medicine (RAAPM) team, which routinely inserts adductor canal catheters pre-operatively for all TKA patients and prescribes inpatient postoperative analgesics.

Maintaining long-term clinical pathway adherence can be challenging for many reasons and pathways should be adaptable. With the hiring of a new surgeon who does not perform LIA and who is a strong supporter of regional anesthesia, the RAAPM team introduced a new ultrasound-guided procedure: the Infiltration between the Popliteal Artery and Capsule of the Knee (IPACK) block [7,8], for controlling posterior knee pain in the new surgeon's TKA patients as a quality improvement project to ensure consistency in care among TKA patients at our facility. We retrospectively compared postoperative outcomes before and after implementation of IPACK blocks, hypothesizing that post-IPACK patients will report lower pain scores on postoperative day (POD) 0 than pre-IPACK patients. We also describe the process of implementing this clinical practice change.

\section{Materials and Methods}

This retrospective cohort study was reviewed and approved with a waiver of informed consent by the local Institutional Review Board (Stanford, CA, USA) and Veterans Affairs (VA) Research Committee (Palo Alto, CA, USA).

\section{Setting}

The project was conducted at a tertiary care university-affiliated VHA hospital with a Peri-operative Surgical Home (PSH) model of care [9]. A RAAPM team, staffed by an attending anesthesiologist, RAAPM fellow, and nurse practitioner, perform peri-operative nerve block procedures, directly co-manages orthopedic surgery and neurosurgical spine surgery patients, provides consultation for other hospitalized patients, and are available around the clock $[9,10]$.

\section{Implementation}

This implementation of a clinical practice change was designed as a quality improvement project using 1 cycle of a plando-study-act [11]. The steps were planned in advance with various stakeholder groups including RAAPM anesthesiologists, orthopedic surgeons, and nurses in the post-anesthesia care unit (PACU) and surgical ward. A need for an infiltrative technique was identified and options included LIA administered by the new surgeon or IPACK blocks performed by the RAAPM team. A collective decision was made for the RAAPM team to provide pre-operative IPACK blocks as part of the TKA clinical pathway with strong support from the new surgeon who favored this approach due to the timing (prior to insertion of implants) and image guidance (ultrasound). The IPACK technique was researched and disseminated to RAAPM team members, and the project was initiated in May 2018 for all knee arthroplasty patients of this single surgeon. All other TKA patients continued to receive surgeon-administered LIA.

For all patients undergoing knee arthroplasty surgery, the RAAPM team placed an ultrasound-guided adductor canal catheter (ACC) pre-operatively using a technique described previously [12]. In the IPACK implementation cohort, after the ACC insertion, the IPACK block was performed under ultrasound guidance using a sterile technique with the operative extremity cleansed from the inguinal ligament down to the popliteal crease. Using the technique originated by Dr. Sanjay Sinha (personal communication), the leg was positioned with the knee flexed and hip externally rotated. A high-frequency ultrasound transducer (HFL50, FUJIFILM Sonosite, USA) was used to visualize the popliteal artery and posterior surface of the distal femur (Fig. 1). If the femoral condyles were seen, the transducer was moved proximally to a position over the distal femoral shaft. Using an in-plane approach, an $8.9 \mathrm{~cm} 20$-gauge Tuohy-tip needle (Epimed, USA) was inserted from medial to lateral to position the needle tip between the popliteal artery and the femur (Fig. 1). Twenty $\mathrm{ml}$ of $0.2 \%$ ropivacaine was injected incrementally into this space.

The preferred intra-operative anesthetic technique for TKA patients was spinal anesthesia [13], but the choice was ultimately made by the operating room anesthesiologist, patient, and surgeon. A standard tri-compartment knee arthroplasty was performed through a medial parapatellar incision under tourniquet control.

After surgery and upon arrival to the PACU, the ACC perineural infusion of $0.2 \%$ ropivacaine with $6 \mathrm{ml} / \mathrm{h}$ basal infusion and $5 \mathrm{ml}$ on-demand bolus with a $30 \mathrm{~min}$ lockout was initiated (ON-Q Pump with Select-A-Flow Variable Rate and ONDEMAND, Avanos, USA). The RAAPM team assumed primary responsibility for managing postoperative analgesics and rounded 

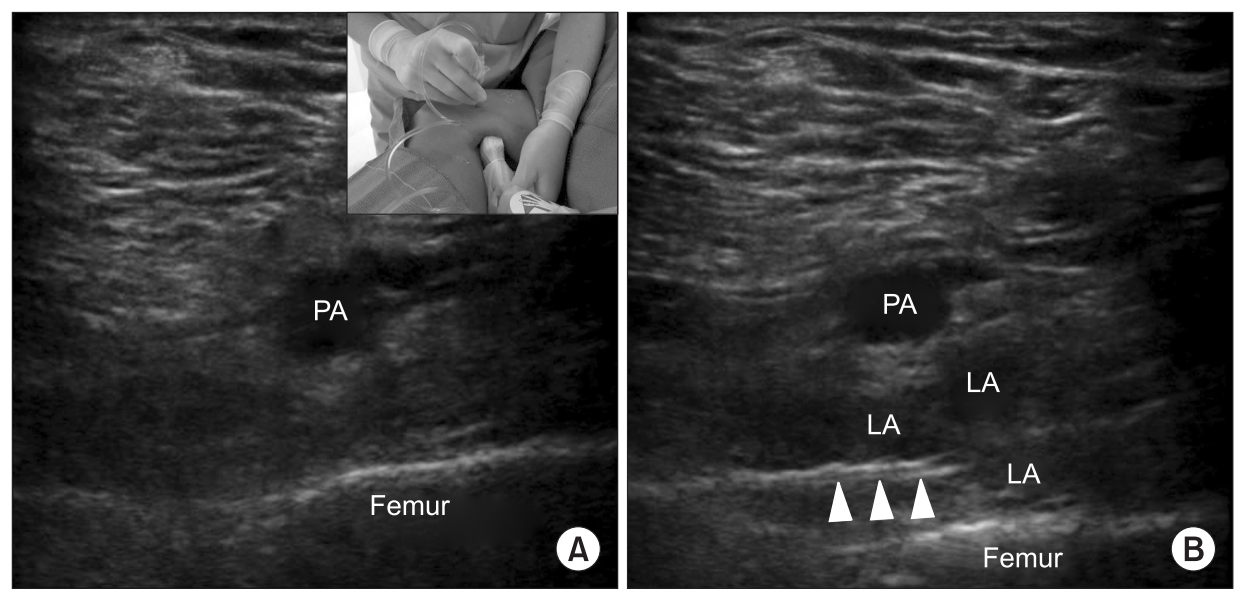

Fig. 1. Ultrasound images demonstrating relevant sono-anatomy for the Infiltration between the Popliteal Artery and Capsule of the Knee (IPACK) before (A) and after (B) local anesthetic injection; PA: popliteal artery, LA: local anesthetic. Arrows indicate the distal end of the needle. on patients daily to assess pain control, rehabilitation progress, and catheter function [6]. Patients began physical therapy (PT) on the morning of POD 1. Adductor canal catheters were typically removed on POD 2. Patients were followed-up in person and/or by phone until the nerve block resolved.

\section{Outcomes}

Data were collected for consecutive TKA patients 4 months post-IPACK implementation (POST) and compared to those for a historical cohort comprised of consecutive TKA patients by the same surgeon 4 months prior to IPACK implementation (PRE). Data normally collected during the postoperative course related to pain, medication administration, and rehabilitation were included and retrospectively reviewed. Baseline characteristics included: age, sex, height, weight, body mass index, American Society of Anesthesiologists physical status, pre-operative opioid prescription (any active opioid prescription within the previous year), case length (out-of-room time minus inroom time), and anesthesia type. Since LIA is only expected to last approximately $12 \mathrm{~h} \mathrm{[2],} \mathrm{the} \mathrm{primary} \mathrm{outcome} \mathrm{for} \mathrm{this} \mathrm{study}$ was patient-reported pain scores (numeric rating scale; $0=$ no pain; $10=$ worst possible pain) on POD 0 . POD 0 data included PACU and ward measures on the surgical date; the number of ward measures varied depending on the time of admission to the ward. Pain was assessed at least once every $4 \mathrm{~h}$ per nursing protocol as well as before and after each as-needed analgesic administration. Daily pain scores were broken down into the lowest reported pain score and the highest reported pain score. Other outcomes included opioid consumption per POD in morphine mg equivalents (MME) with POD 0 including PACU stay; lowest and highest pain scores on POD 1 and 2; length of stay; discharge disposition; maximum ambulation distance on POD 1 and 2; and 30-day adverse events such as new peripheral neuropathies, emergency room visits, hospital readmissions, and major organ system complications.

\section{Statistical analysis}

Statistical analysis was performed using NCSS Statistical Software (NCSS, LLC, USA) and IBM SPSS Statistics Version 25 (IBM Corp., USA). Normality of distribution was determined for all scale variables using the Kolmogorov-Smirnov test. For normally distributed data, single comparisons were performed using the Student's $t$-test; for continuous data in non-normal distributions, the Mann-Whitney $U$ test was used. The chisquared test or Fisher's exact test ( $\mathrm{n}<5$ in any field) was used for comparisons of categorical data. For all comparisons, $\mathrm{P}<0.05$ was considered statistically significant.

\section{Results}

Two-hundred thirteen patients underwent knee replacements in the 8-month study period (February through September 2018); 4 months PRE and 4 months POST. In 125 patients, LIA was performed by the surgeon. Of the remaining 88 patients, 8 patients were excluded ( 3 uni-compartmental arthroplasty patients, 3 revision TKA patients, and 2 patients in the POST group [4\%] who did not receive an IPACK block after implementation of the new protocol). The final cohort consisted of 80 patients ( $n=32$ for PRE and $n=48$ for POST). There were no differences between groups in terms of demographic and baseline characteristics (Table 1). In the POST group, 16/48 (33\%) patients were considered pre-operative opioid users compared to $19 / 32(59 \%)$ in the PRE group $(\mathrm{P}=0.021)$ based on having an active prescription for an opioid within 1 year of surgery. Twenty-eight out of 32 (88\%) PRE patients and 33 out of 48 (69\%) POST patients had spinal anesthesia which was similar between groups $(\mathrm{P}=0.064)$. 


\section{Primary outcome}

On POD 0, the lowest patient-reported pain score (median [10th-90th percentiles]) was lower for the POST group than for the PRE group (0 [0-4.3] vs. $2.5[0-7] ; \mathrm{P}=0.003)$. The highest patient-reported pain scores on POD 0 and the lowest and highest pain scores on POD 1 and 2 were similar between the groups (Fig. 2).

\section{Secondary outcomes}

In the PACU, overall opioid consumption in MME (median [10th-90th percentiles]) was 4 (0-65) for the PRE group and $0(0-62)$ for the POST group $(\mathrm{P}=0.584)$. Sixteen PRE patients (50\%) and 26 POST patients (54\%) required no opioids in the PACU $(\mathrm{P}=0.715)$. The lowest pain score recorded in the PACU was $0(0-5)$ for the PRE group and $0(0-4)$ for the POST group $(\mathrm{P}=0.444)$. The highest pain score recorded in the PACU was

Table 1. Demographics and Baseline Characteristics

\begin{tabular}{lccr}
\hline & $\begin{array}{c}\text { Pre-IPACK } \\
(\mathrm{n}=32)\end{array}$ & $\begin{array}{c}\text { Post-IPACK } \\
(\mathrm{n}=48)\end{array}$ & P value \\
\hline Age $(\mathrm{yr})$ & $68(56-73)$ & $70(59-79)$ & 0.107 \\
Sex $(\mathrm{F})$ & $2(6 \%)$ & $4(8 \%)$ & $>0.999$ \\
Height $(\mathrm{cm})$ & $175(163-183)$ & $174(165-183)$ & 0.608 \\
Weight $(\mathrm{kg})$ & $95(71-118)$ & $94(77-114)$ & 0.831 \\
Body mass index $\left(\mathrm{kg} / \mathrm{m}^{2}\right)$ & $30(24-40)$ & $32(25-39)$ & 0.674 \\
ASA physical status & $3(2-3)$ & $3(2-3)$ & 0.383 \\
Case length (min) & $203(171-234)$ & $204(163-239)$ & 0.410 \\
\hline
\end{tabular}

Values are presented as median (10th-90th percentiles) or number (\%), as applicable. IPACK: Infiltration between the Popliteal Artery and Capsule of the Knee, ASA: American Society of Anesthesiologists, Case Length: out of room time - in room time.

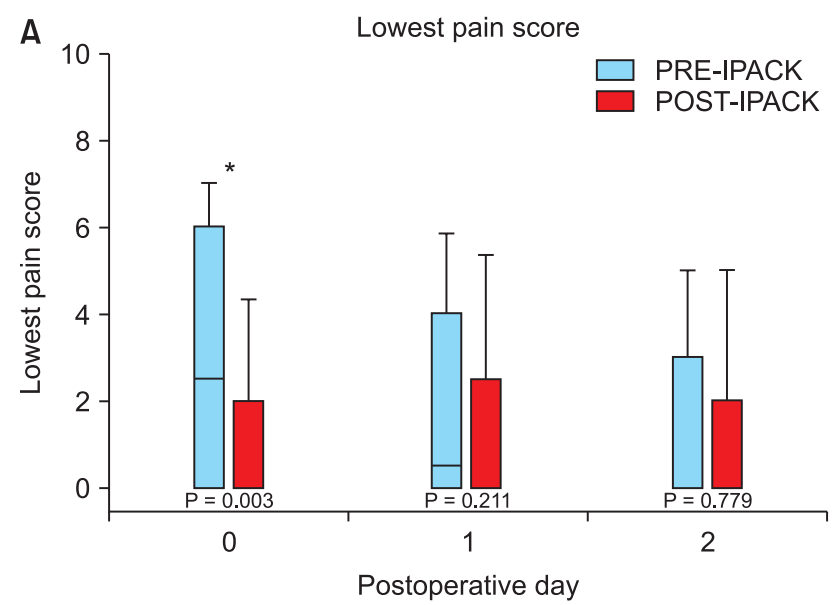

$4(0-10)$ for the PRE group and $1(0-9)$ for the POST group $(P=0.597)$. Since the anesthetic technique could be expected to influence PACU outcomes, we analyzed subgroups based on anesthetic technique. For patients who received spinal anesthesia, the PACU opioid consumption in MME was 0 (0-66) for the PRE group $(\mathrm{n}=28)$ and $0(0-51)$ for the POST group $(\mathrm{n}=33$; $\mathrm{P}=$ $0.168)$; the lowest reported pain was $0(0-5)$ for the PRE group and $0(0-5)$ for the POST group $(\mathrm{P}=0.976)$; and the highest reported pain was $1.5(0-10)$ for the PRE group and $0(0-8)$ for the POST group $(\mathrm{P}=0.175)$. For patients who did not receive spinal anesthesia, PACU opioid consumption in MME was 22 (4-55) for the PRE group $(\mathrm{n}=4)$ and $23(0-72)$ for the POST group $(\mathrm{n}=$ $15 ; \mathrm{P}=0.880)$; the lowest reported pain was 0 (0-4.2) for the PRE group and $2(0-4)$ for the POST group $(\mathrm{P}=0.568)$; and the highest reported pain was $5.5(1.5-7.4)$ for the PRE group and 7.5 $(0.6-10)$ for the POST group $(\mathrm{P}=0.420)$. There were no differences in the rates of postoperative adverse events (Table 2).

On POD 0, the total opioid consumption in MME was 47 (15-100) for the PRE group and 34 (13-93) for the POST group $(\mathrm{P}=0.166)$. On POD 1 , the total opioid consumption was 48

Table 2. Secondary Outcomes

\begin{tabular}{lccr}
\hline & $\begin{array}{c}\text { Pre-IPACK } \\
(\mathrm{n}=32)\end{array}$ & $\begin{array}{c}\text { Post-IPACK } \\
(\mathrm{n}=48)\end{array}$ & P value \\
\hline Length of stay (days) & $3(2-4)$ & $3(2-5)$ & 0.690 \\
Discharge to home & $30(94 \%)$ & $42(88 \%)$ & 0.466 \\
New peripheral neuropathy & $0(0 \%)$ & $0(0 \%)$ & $>0.999$ \\
Emergency room visit only & $2(6 \%)$ & $3(6 \%)$ & $>0.999$ \\
Hospital readmission & $0(0 \%)$ & $1(2 \%)$ & $>0.999$ \\
Major adverse events & $0(0 \%)$ & $1(2 \%)$ & $>0.999$ \\
\hline
\end{tabular}

Values are presented as median (10th-90th percentiles) or number (\%), as applicable. IPACK: Infiltration between the Popliteal Artery and Capsule of the Knee.

Fig. 2. Lowest (A) and highest (B) pain scores reported by patients in the PRE- and POST-IPACK groups on postoperative days 0 , 1, and 2 using a numeric rating scale. IPACK: Infiltration between the Popliteal Artery and Capsule of the Knee. ${ }^{*} \mathrm{P}<0.05$.

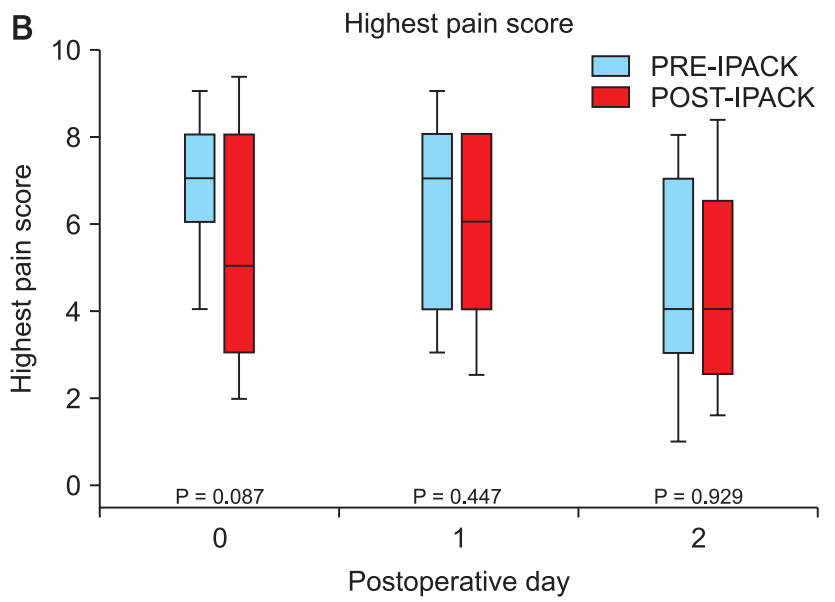


(23-113) for the PRE group and 45 (15-85) for the POST group $(\mathrm{P}=0.150)$. On POD 2, the total opioid consumption was 38 (090) for the PRE group and 26 (5-72) for the POST group ( $\mathrm{P}=0.265)$. On POD 1, the ambulation distance in meters was 23 (1-60) for the PRE group and $15(0-47)$ for the POST group ( $\mathrm{P}=0.410)$. On POD 2, the ambulation distance was 46 (4-61) for the PRE group and 46 (10-61) for the POST group $(\mathrm{P}=0.886)$.

\section{Discussion}

Although the results of our study show relatively limited benefits in terms of pain scores from adding IPACK blocks within a robust multimodal analgesic regimen, this process demonstrates the feasibility of implementing practice changes in an efficient manner. Revision of a multimodal analgesic clinical pathway to incorporate IPACK blocks for TKA patients in our practice occurred rapidly and with very high adherence (96\%). In addition, increasing patient access to non-opioid analgesia in the form of a local anesthetic and decreasing disparities in pain management between TKA patients within a single institution arguably justified our adoption of IPACK blocks [14].

The "ideal" regional analgesic for TKA patients continues to evolve, and providing optimal pain control must be balanced with potentially competing goals like early mobility [1]. Modern clinical pathways for joint replacement surgeries increasingly incorporate non-opioid systemic analgesics such as acetaminophen, non-steroidal anti-inflammatory drugs, and local anesthetics $[6,15,16]$. In this context, minor variations in the mode of local anesthesia delivery like the addition of IPACK blocks in a practice routinely providing continuous adductor canal blocks for TKA patients $[6,12]$, may not result in major changes in clinical outcomes. Of note, TKA patients at our institution do not begin physical therapy until POD 1; thus, any potential rehabilitative advantages due to IPACK's analgesic effects on POD 0 remain unknown since local infiltration around the knee does not reliably last more than $12 \mathrm{~h} \mathrm{[2].} \mathrm{To} \mathrm{date,} \mathrm{there} \mathrm{are} \mathrm{few} \mathrm{pub-}$ lished studies evaluating the IPACK block $[7,8,17,18]$, of which only 2 are prospective $[8,17]$. In a randomized clinical trial by Kim et al. [8], patients received either surgeon-administered LIA alone or a combined intervention consisting of an IPACK block with up to $40 \mathrm{ml}$ of $0.25 \%$ bupivacaine, a single-injection adductor canal block (ACB), and a modified surgeon-administered LIA. While pain scores in the immediate postoperative period favored the combined local anesthetic intervention (IPACK) group, opioid reduction did not persist beyond the first $24 \mathrm{~h}$ and there were no differences in physical therapy outcomes [8]. The study by Sankineani et al. [17], was a prospective, non-randomized open label study comparing IPACK blocks using 15 $\mathrm{ml}$ of $0.2 \%$ ropivacaine plus a single-injection $\mathrm{ACB}$ and $\mathrm{ACB}$ alone. The IPACK-ACB group demonstrated lower pain scores on POD 0-2 and greater physical function vs. the ACB group although pain scores were low overall in both groups and the multimodal regimen was limited to acetaminophen $3 \mathrm{~g}$ total and gabapentin $300 \mathrm{mg}$ per day [17].

Our study is the first to evaluate the addition of the IPACK block to a continuous adductor canal block within a multimodal analgesic clinical pathway including multiple classes of analgesic interventions. This clinical pathway at our institution is mature and is founded on the use of continuous peripheral nerve blocks [6]. Over the past 8 years, individual elements of the pathway have been revised to make perineural infusions more selective [19], expand the use of non-opioid analgesics [15], make spinal anesthesia the preferred intra-operative anesthetic technique [13], and provide high quality education for patients that can help them participate in their own pain management [20]. This may, at least partially, explain why the results of the present study are not strongly positive compared to those of previously published studies.

The primary reason for implementing the IPACK block was to provide consistent care among TKA patients at a single institution, which took place over the course of several weeks. The process of implementing the clinical practice change in the face of new evidence has been estimated to take 17 years [21]. Reasons for this delay in translating evidence to practice are attributed to a lack of familiarity, lack of awareness, lack of agreement, lack of outcome expectancy, lack of self-efficacy, lack of motivation, and external barriers [22]. A culture supportive of rapid changes in clinical practice has been demonstrated previously at our institution [13]. The collaborative Peri-operative Surgical Home (PSH) model with co-management of orthopedic surgery patients by surgeons and RAAPM anesthesiologists supports incremental improvements in the care of patients $[9,13]$. We cannot say for certain why there is a difference in pre-operative opioid usage between the 2 groups in our study with a lower rate in the POST group, but we speculate that changes in prescribing practices as a result of the ongoing opioid epidemic [23] may be at least partially responsible.

This study has several limitations. First, this was a single-center retrospective study with a small sample size. We attempted to minimize bias by including all consecutive TKA patients in the initial sample. Secondly, certain institutional factors may limit generalizability. Our institution is a university-affiliated VA hospital serving only veterans and this population is mostly male with a high prevalence of multiple comorbidities [24]; therefore, results may be different in the general population. In addition, the PSH model is not employed at every institution and likely promotes rapid process improvement. Thirdly, TKA patients at our hospital do not begin physical therapy until POD 1 due to staffing limitations. Therefore, any pro-mobilization benefits from posterior knee analgesia due to the IPACK block on POD 
0 remain unrealized since the block resolves before the first physical therapy consult on POD 1. Finally, the optimal dosing regimen for the IPACK block is still unknown. While our dose of ropivacaine $(20 \mathrm{ml}$ of $0.2 \%$ ) is similar to the dose used in 1 prospective study [17], other studies used higher doses [7,8]. One study of 10 fresh frozen cadaver knees using $20 \mathrm{ml}$ of colored latex solution in an IPACK injection has shown potential spread to the terminal branches of the sciatic nerve, which may suggest limiting the volume of the injectate to $20 \mathrm{ml}$ or less [18].

In conclusion, our study showed minimal short-term benefits of adding an IPACK block to a continuous adductor canal block within a multimodal analgesic clinical pathway after primary unilateral TKA. We also demonstrated the feasibility of rapidly adapting an existing clinical pathway in the face of changing conditions to provide consistent patient care.

\section{Conflicts of Interest}

No potential conflict of interest relevant to this article was reported.

\section{Author Contributions}

Brandon Kandarian (Conceptualization; Data curation; Methodology; Writing - original draft; Writing - review \& editing)

Pier F. Indelli (Conceptualization; Investigation; Methodology; Project administration; Writing - original draft; Writing - re- view \& editing)

Sanjay Sinha (Conceptualization; Investigation; Methodology; Writing - original draft; Writing - review \& editing)

Oluwatobi O. Hunter (Conceptualization; Data curation; Methodology; Writing - review \& editing)

Rachel R. Wang (Methodology; Resources; Validation; Writing - review \& editing)

T. Edward Kim (Conceptualization; Resources; Validation; Writing - review \& editing)

Alex Kou (Conceptualization; Methodology; Resources; Validation; Writing - review \& editing)

Edward R. Mariano (Conceptualization; Data curation; Formal analysis; Investigation; Methodology; Project administration; Resources; Supervision; Validation; Visualization; Writing original draft; Writing - review \& editing)

\section{ORCID}

Brandon Kandarian, https://orcid.org/0000-0001-5264-9264

Pier F. Indelli, https://orcid.org/0000-0002-4752-8027

Sanjay Sinha, https://orcid.org/0000-0002-9955-0605

Oluwatobi O. Hunter, https://orcid.org/0000-0002-1502-9522

Rachel R. Wang, https://orcid.org/0000-0002-3481-0699

T. Edward Kim, https://orcid.org/0000-0002-1183-3556

Alex Kou, https://orcid.org/0000-0003-1807-1176

Edward R. Mariano, https://orcid.org/0000-0003-2735-248X

\section{References}

1. Webb CA, Mariano ER. Best multimodal analgesic protocol for total knee arthroplasty. Pain Manag 2015; 5: 185-96.

2. Kehlet H, Andersen LØ. Local infiltration analgesia in joint replacement: the evidence and recommendations for clinical practice. Acta Anaesthesiol Scand 2011; 55: 778-84.

3. Tripuraneni KR, Woolson ST, Giori NJ. Local infiltration analgesia in TKA patients reduces length of stay and postoperative pain scores. Orthopedics 2011; 34: 173

4. Perlas A, Kirkham KR, Billing R, Tse C, Brull R, Gandhi R, et al. The impact of analgesic modality on early ambulation following total knee arthroplasty. Reg Anesth Pain Med 2013; 38: 334-9.

5. Kampitak W, Tanavalee A, Ngarmukos S, Amarase C, Apihansakorn R, Vorapalux P. Does adductor canal block have a synergistic effect with local infiltration analgesia for enhancing ambulation and improving analgesia after total knee arthroplasty? Knee Surg Relat Res 2018; 30: $133-41$

6. Steckelberg RC, Funck N, Kim TE, Walters TL, Lochbaum GM, Memtsoudis SG, et al. Adherence to a multimodal analgesic clinical pathway: a within-group comparison of staged bilateral knee arthroplasty patients. Reg Anesth Pain Med 2017; 42: 368-71.

7. Thobhani S, Scalercio L, Elliott CE, Nossaman BD, Thomas LC, Yuratich D, et al. Novel regional techniques for total knee arthroplasty promotereduced hospital length of stay: an analysis of 106 patients. Ochsner J 2017; 17: 233-8.

8. Kim DH, Beathe JC, Lin Y, YaDeau JT, Maalouf DB, Goytizolo E, et al. Addition of infiltration between the popliteal artery and the capsule of the posterior knee and adductor canal block to periarticular injection enhances postoperative pain control in total knee arthroplasty: a randomized controlled trial. Anesth Analg 2018. Advance Access published on Sep 12, 2018. doi: 10.1213/ANE.0000000000003794.

9. Walters TL, Howard SK, Kou A, Bertaccini EJ, Harrison TK, Kim TE, et al. Design and implementation of a perioperative surgical home at a veterans affairs hospital. Semin Cardiothorac Vasc Anesth 2016; 20: 133-40.

10. Mariano ER, Walters TL, Kim TE, Kain ZN. Why the perioperative surgical home makes sense for veterans affairs health care. Anesth Analg 2015; 120: 1163-6. 
11. Taylor MJ, McNicholas C, Nicolay C, Darzi A, Bell D, Reed JE. Systematic review of the application of the plan-do-study-act method to improve quality in healthcare. BMJ Qual Saf 2014; 23: 290-8.

12. Mariano ER, Kim TE, Wagner MJ, Funck N, Harrison TK, Walters T, et al. A randomized comparison of proximal and distal ultrasoundguided adductor canal catheter insertion sites for knee arthroplasty. J Ultrasound Med 2014; 33: 1653-62.

13. Mudumbai SC, Walters TL, Howard SK, Kim TE, Lochbaum GM, Memtsoudis SG, et al. The Perioperative Surgical Home model facilitates change implementation in anesthetic technique within a clinical pathway for total knee arthroplasty. Healthc (Amst) 2016; 4: 334-9.

14. Mudumbai SC, Auyong DB, Memtsoudis SG, Mariano ER. A pragmatic approach to evaluating new techniques in regional anesthesia and acute pain medicine. Pain Manag 2018; 8: 475-85.

15. Schwenk ES, Mariano ER. Designing the ideal perioperative pain management plan starts with multimodal analgesia. Korean J Anesthesiol 2018; 71: 345-52.

16. Memtsoudis SG, Poeran J, Zubizarreta N, Cozowicz C, Mörwald EE, Mariano ER, et al. Association of multimodal pain management strategies with perioperative outcomes and resource utilization: a population-based study. Anesthesiology 2018; 128: 891-902.

17. Sankineani SR, Reddy AR, Eachempati KK, Jangale A, Gurava Reddy AV. Comparison of adductor canal block and IPACK block (interspace between the popliteal artery and the capsule of the posterior knee) with adductor canal block alone after total knee arthroplasty: a prospective control trial on pain and knee function in immediate postoperative period. Eur J Orthop Surg Traumatol 2018; 28: 1391-5.

18. Niesen AD, Harris DJ, Johnson CS, Stoike DE, Smith HM, Jacob AK, et al. Interspace between popliteal artery and posterior capsule of the knee (IPACK) injectate spread: a cadaver study. J Ultrasound Med 2019; 38: 741-5.

19. Mudumbai SC, Kim TE, Howard SK, Workman JJ, Giori N, Woolson S, et al. Continuous adductor canal blocks are superior to continuous femoral nerve blocks in promoting early ambulation after TKA. Clin Orthop Relat Res 2014; 472: 1377-83.

20. Yajnik M, Hill JN, Hunter OO, Howard SK, Kim TE, Harrison TK, et al. Patient education and engagement in postoperative pain management decreases opioid use following knee replacement surgery. Patient Educ Couns 2019; 102: 383-7.

21. Morris ZS, Wooding S, Grant J. The answer is 17 years, what is the question: understanding time lags in translational research. J R Soc Med 2011; 104: 510-20.

22. Cabana MD, Rand CS, Powe NR, Wu AW, Wilson MH, Abboud PA, et al. Why don't physicians follow clinical practice guidelines? A framework for improvement. JAMA 1999; 282: 1458-65.

23. Hill MV, Stucke RS, McMahon ML, Beeman JL, Barth RJ Jr. An educational intervention decreases opioid prescribing after generalsurgical operations. Ann Surg 2018; 267: 468-72.

24. Singh JA, Sloan J. Higher comorbidity, poor functional status and higher health care utilization in veterans with prevalent total knee arthroplasty or total hip arthroplasty. Clin Rheumatol 2009; 28: 1025-33. 